\title{
ORIGINAL
}

\section{Identification and validation of biomarkers of persistent acute kidney injury: the RUBY study}

Eric Hoste ${ }^{1}$, Azra Bihorac ${ }^{2}$, Ali Al-Khafaji ${ }^{3}$, Luis M. Ortega ${ }^{4}$, Marlies Ostermann ${ }^{5}$, Michael Haase ${ }^{6,7}$, Kai Zacharowski ${ }^{8}$, Richard Wunderink ${ }^{9}$, Michael Heung ${ }^{10}$, Matthew Lissauer ${ }^{11}$, Wesley H. Self ${ }^{12}$, Jay L. Koyner ${ }^{13}$, Patrick M. Honore ${ }^{14}$, John R. Prowle ${ }^{15}$, Michael Joannidis ${ }^{16}$, Lui G. Forni ${ }^{17}$, J. Patrick Kampf ${ }^{18}$, Paul McPherson ${ }^{18}$, John A. Kellum ${ }^{19}$ and Lakhmir S. Chawla ${ }^{20^{*}}$ (D) on behalf of the RUBY Investigators

(C) 2020 The Author(s)

\begin{abstract}
Purpose: The aim of the RUBY study was to evaluate novel candidate biomarkers to enable prediction of persistence of renal dysfunction as well as further understand potential mechanisms of kidney tissue damage and repair in acute kidney injury (AKI).

Methods: The RUBY study was a multi-center international prospective observational study to identify biomarkers of the persistence of stage $3 \mathrm{AKI}$ as defined by the KDIGO criteria. Patients in the intensive care unit (ICU) with moderate or severe AKI (KDIGO stage 2 or 3 ) were enrolled. Patients were to be enrolled within $36 \mathrm{~h}$ of meeting KDIGO stage 2 criteria. The primary study endpoint was the development of persistent severe AKI (KDIGO stage 3) lasting for $72 \mathrm{~h}$ or more (NCT01868724).

Results: 364 patients were enrolled of whom 331 (91\%) were available for the primary analysis. One hundred ten (33\%) of the analysis cohort met the primary endpoint of persistent stage 3 AKI. Of the biomarkers tested in this study, urinary $\mathrm{C}-\mathrm{C}$ motif chemokine ligand 14 (CCL14) was the most predictive of persistent stage $3 \mathrm{AKI}$ with an area under the receiver operating characteristic curve (AUC) $(95 \% \mathrm{Cl})$ of $0.83(0.78-0.87)$. This AUC was significantly greater than values for other biomarkers associated with AKI including urinary KIM-1, plasma cystatin $C$, and urinary NGAL, none of which achieved an AUC $>0.75$.
\end{abstract}

Conclusion: Elevated urinary CCL14 predicts persistent AKI in a large heterogeneous cohort of critically ill patients with severe AKI. The discovery of CCL14 as a predictor of persistent AKI and thus, renal non-recovery, is novel and could help identify new therapeutic approaches to AKI.

Keywords: Persistent acute kidney injury, Biomarkers, C-C motif chemokine ligand 14 (CCL14), NGAL (Neutrophil gelatinase-associated lipocalin), Plasma cystatin C, KIM-1 (kidney injury molecule-1)

\footnotetext{
*Correspondence: minkchawla@gmail.com

${ }^{20}$ Department of Medicine, Veterans Affairs Medical Center, 3350 La Jolla

Village Dr, San Diego, CA 92161, USA
}

Full author information is available at the end of the article

The members of the RUBY Investigators are listed in Acknowledgements.

\section{实 Springer}




\section{Introduction}

Acute kidney injury (AKI) is a global problem affecting more than $10 \%$ of all hospitalized patients and approaching two-thirds of those admitted to intensive care units, with survival related not only to the severity, but also to the duration of renal dysfunction [1-3]. Recent evidence suggests that two-thirds of patients with AKI resolve their renal dysfunction within 3-7 days whereas those who persist have dramatically reduced survival over the following year [4]. Persistence of AKI is of grave importance in that it increases an individual's risk of developing chronic kidney disease (CKD) and the consequences thereof [5]. This link between AKI and CKD has been established over the last decade and specific recommendations for the management of patients with AKI have been proposed in order to potentially influence this transition $[5,6]$. It follows that early identification of individuals at risk of persistent AKI would enable appropriate delivery of these proposed interventions, but also may identify individuals where newer therapies to attenuate AKI could be targeted.

Not only is persistence of AKI relevant to longer term outcomes, clinical decision-making is also critically affected by physician expectations surrounding renal recovery and the decision of when to initiate renal replacement therapy (RRT). Currently this is almost totally dependent on clinical expectations as to the likelihood of recovery with no available diagnostics to aid this decision process. As such, significant controversy exists around the timing of RRT with studies showing that some patients can benefit from the earlier initiation of RRT, while other studies demonstrate that some individuals receive RRT who may not require such treatment as they will recover renal function soon [7, 8]. Early and reliable identification of those who will recover renal function may enable a more stratified approach to management, thus avoiding the incumbent risks of extracorporeal therapy.

Of equal or even greater importance is that the mechanisms responsible for recovery from AKI are incompletely understood. Recovery following AKI involves dedifferentiation and proliferation of viable cells which then re-epithelialize the nephron. Failure to regenerate lost cells results in a maladaptive repair and fibrosis [9]. Key mechanisms and triggers for these processes are largely unknown.

In order to find potential markers for persistent AKI, both as candidates for diagnostic testing as well as potential drug targets, we tested novel candidate biomarkers in an international multi-center prospective study to predict persistence of AKI.

\section{Take-Home Message}

This manuscript is the first report on a new biomarker, urinary chemokine ligand CCL14, which is a predictor of persistent stage $3 \mathrm{AKI}$. CCL14 has never been identified in the pathobiology of AKI before. The role of this chemokine is entirely consistent with what is known about the development of renal damage and repair, in particular macrophage trafficking and subsequent fibrosis.

\section{Methods}

\section{Study design}

The RUBY study is an international prospective observational study aimed at identifying biomarkers associated with the persistence of stage $3 \mathrm{AKI}$ as defined by the KDIGO criteria [10]. Adult patients in the intensive care unit (ICU) with moderate or severe AKI (stage 2 or 3) and an indwelling urinary catheter were enrolled from June 2013 through May 2014. Both serum creatinine and urine output criteria were used to determine KDIGO AKI stage [10]. Patients were enrolled within $36 \mathrm{~h}$ of meeting the KDIGO criteria as determined by the investigator based on serum creatinine and urine output data available at the time of enrollment. Any patients later determined not to be at stage 2 or 3 at enrollment by retrospective analysis were retained in the primary analysis cohort based on the intent to diagnose principle [11]. Patients were excluded if they had a prior kidney transplant, were receiving, or were in imminent need of RRT, were receiving comfort measures only, or had known infection with human immunodeficiency virus or active hepatitis. Written informed consent was provided by all patients or their legally authorized representatives prior to enrollment. The RUBY study was approved by the Western Institutional Review Board (Puyallup, WA) and by individual investigational review boards or ethics committees as required by each enrolling site.

The primary study endpoint was the development of persistent, severe AKI (stage 3), lasting for $72 \mathrm{~h}$ or more given that evidence demonstrates that the short and longterm risk of death or need for RRT is greatest for patients who persist with AKI for longer than 3 days [4]. This primary endpoint is consistent with more recent studies on initiation of RRT which specified $72 \mathrm{~h}$ of oliguria as criterion for initiation of treatment [12].

For the purposes of determining the primary endpoint, each patient's baseline serum creatinine was retrospectively adjudicated by a physician blinded to biomarker data based on serum creatinine data collected from the medical record from 6 months prior to enrollment through 3 months after enrollment. Patients not at stage $3 \mathrm{AKI}$ at enrollment were required to reach stage 3 within $48 \mathrm{~h}$ and then persist in stage $3 \mathrm{AKI}$ for at least $72 \mathrm{~h}$ to be considered endpoint positive. Patients who 
had a maximum AKI stage of 3 and died within $48 \mathrm{~h}$ of enrollment were also considered endpoint positive. Additionally, patients with stage 3 AKI who failed to achieve 72 consecutive hours due to initiation of RRT or death were considered endpoint positive (Fig. 1). The secondary endpoints were the composite of RRT initiation or death within 90 days and major adverse kidney events at 90 $( \pm 7)$ days $\left(\mathrm{MAKE}_{90}\right)$ [13]. MAKE was defined as the composite of $\geq 25 \%$ loss in estimated glomerular filtration rate (eGFR), dialysis, or death. Estimated GFR was calculated from serum creatinine using the MDRD equation.

\section{Sample and data collection}

Blood and urine samples were collected at enrollment and centrifuged. Plasma (EDTA), serum, and urine supernatants were frozen within $2 \mathrm{~h}$ of sample collection, stored at $\leq-70{ }^{\circ} \mathrm{C}$, and thawed immediately prior to analysis. Patient data abstracted from the medical record included demographics, medical history, APACHE III variables, reason for admission, hourly urine output from the day prior to enrollment through study day 7, as well as serum creatinine, RRT, and death through 90 days after enrollment. Telephone follow-up was conducted at $90( \pm 7)$ days to provide additional data for the $\mathrm{MAKE}_{90}$ endpoint.

\section{Biomarker identification and testing}

Potential candidate biomarkers from proteins associated with apoptosis, necrosis, endothelial injury, cell-cell and cell-matrix adhesion, cytoprotection, oxidative processes, cell-cycle regulation, inflammation, tubular injury, immune function, and fibrosis were selected for biologic plausibility based on previously described methods [14]. The aim was to determine whether the candidate proteins could aid in risk assessment for persistence of renal dysfunction as well as contribute to the understanding behind the mechanisms of renal tissue damage and repair. A broad set of potential biomarkers were assessed focused on potential mechanisms of action as opposed to relying on defined AKI biomarkers alone.

Biomarkers were measured in urine and/or plasma at Astute Medical (San Diego, CA, USA) by technicians blinded to clinical data. Plasma and urinary NGAL were measured by enzyme-linked immunosorbent assay (Bioporto, Copenhagen, Denmark). The combination of tissue inhibitor of metallproteinases-2 (TIMP-2) and insulin-like growth factor-binding protein 7 (IGFBP7) was measured in urine with the NEPHROCHECK CCL14 Test (Astute Medical) as was the final analysis of urinary $\mathrm{C}-\mathrm{C}$ motif chemokine ligand 14 (CCL14) using a prototype clinical immunoassay. Proenkephalin 119-159 was measured in plasma with sphingotest pro-ENK (sphingotec GmBH, Hennigsdorf, Germany). Other previously described biomarkers of AKI were measured on the Luminex 200 platform (Luminex, Austin, TX, USA) either with commercial kits from EMD Millipore (Billerica, MA, USA) [urinary chitinase-3-like

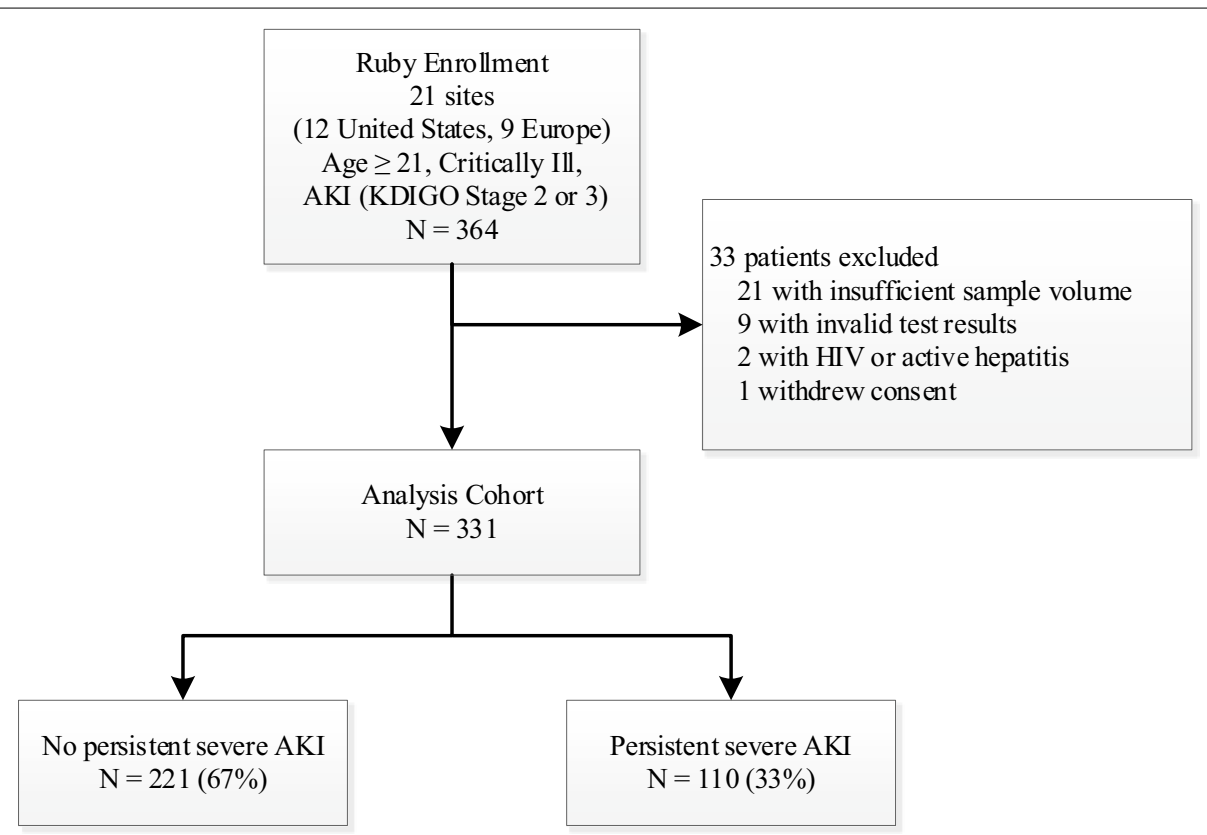

Fig. 1 Patient flow diagram. After exclusions, 331 patients with urinary CCL14 results were available for analysis. 110 patients met the persistent severe AKI endpoint (at least 72 consecutive hours of stage 3 AKI, initiation of dialysis, or death following stage 3 AKI) 
Table 1 Baseline patient characteristics for all patients and by AKI persistence status

\begin{tabular}{|c|c|c|c|c|}
\hline & All patients & Not persistent severe AKI & Persistent severe AKI & $p$ value \\
\hline Patients & 331 & 221 & 110 & \\
\hline Male & $207(62.5 \%)$ & $136(61.5 \%)$ & $71(64.5 \%)$ & 0.631 \\
\hline Age (years) & $64(55-73)$ & $64(54-73)$ & $64(55-71)$ & 0.636 \\
\hline Body mass index $\left(\mathrm{kg} / \mathrm{m}^{2}\right)$ & $29(25-35)$ & $30(26-36)$ & $28(25-34)$ & 0.013 \\
\hline Race & & & & 0.371 \\
\hline Black or African American & $34(10.3 \%)$ & $26(11.8 \%)$ & $8(7.3 \%)$ & \\
\hline Other/unknown & $17(5.1 \%)$ & $10(4.5 \%)$ & $7(6.4 \%)$ & \\
\hline White or caucasian & $280(84.6 \%)$ & $185(83.7 \%)$ & $95(86.4 \%)$ & \\
\hline \multicolumn{5}{|l|}{ Chronic comorbidities } \\
\hline Chronic kidney disease & $58(17.5 \%)$ & $36(16.3 \%)$ & $22(20 \%)$ & 0.444 \\
\hline Diabetes mellitus & 109 (32.9\%) & $82(37.1 \%)$ & $27(24.5 \%)$ & 0.025 \\
\hline Congestive heart failure & $74(22.4 \%)$ & $51(23.1 \%)$ & $23(20.9 \%)$ & 0.677 \\
\hline Coronary artery disease & $117(35.3 \%)$ & $84(38 \%)$ & $33(30 \%)$ & 0.179 \\
\hline Hypertension & $226(68.3 \%)$ & $154(69.7 \%)$ & $72(65.5 \%)$ & 0.454 \\
\hline Chronic obstructive pulmonary disease & $55(16.6 \%)$ & $35(15.8 \%)$ & $20(18.2 \%)$ & 0.639 \\
\hline Cancer & $84(25.4 \%)$ & $57(25.8 \%)$ & $27(24.5 \%)$ & 0.894 \\
\hline \multicolumn{5}{|l|}{ Reason for ICU admission } \\
\hline Respiratory & $95(28.7 \%)$ & $62(28.1 \%)$ & $33(30 \%)$ & 0.797 \\
\hline Surgery & $105(31.7 \%)$ & $74(33.5 \%)$ & $31(28.2 \%)$ & 0.381 \\
\hline Cardiovascular & $148(44.7 \%)$ & $96(43.4 \%)$ & $52(47.3 \%)$ & 0.558 \\
\hline Sepsis & $74(22.4 \%)$ & $49(22.2 \%)$ & $25(22.7 \%)$ & $>0.999$ \\
\hline Neurological & $16(4.8 \%)$ & $12(5.4 \%)$ & $4(3.6 \%)$ & 0.593 \\
\hline Trauma & $7(2.1 \%)$ & $6(2.7 \%)$ & $1(0.9 \%)$ & 0.432 \\
\hline Other & $107(32.3 \%)$ & $74(33.5 \%)$ & $33(30 \%)$ & 0.536 \\
\hline Vasopressors & $210(63.4 \%)$ & $139(62.9 \%)$ & $71(64.5 \%)$ & 0.809 \\
\hline Diuretics & $178(53.8 \%)$ & $114(51.6 \%)$ & $64(58.2 \%)$ & 0.293 \\
\hline Fluid balance (mL) & 3271 (1267-6422) & $2962(1082-6028)$ & $3768(1852-7353)$ & 0.037 \\
\hline Days from ICU admission to enrollment & $1.1(0.7-2.2)$ & $1.1(0.7-2.4)$ & $1.2(0.7-1.9)$ & 0.990 \\
\hline Mechanical ventilation & 185 (55.9\%) & $121(54.8 \%)$ & $64(58.2 \%)$ & 0.560 \\
\hline Baseline serum creatinine (mg/dL) & $1(0.8-1.2)$ & $1(0.8-1.2)$ & $1(0.8-1.3)$ & 0.083 \\
\hline Enrollment serum creatinine $(\mathrm{mg} / \mathrm{dL})$ & $2.4(1.7-3.3)$ & $2.1(1.5-2.8)$ & $3.4(2.6-4.2)$ & $<0.001$ \\
\hline Enrollment KDIGO Stage $\mathrm{e}^{\mathrm{a}}$ & & & & $<0.001$ \\
\hline No AKI & $14(4.2 \%)$ & $14(6.3 \%)$ & $0(0 \%)$ & \\
\hline Stage 1 & $39(11.8 \%)$ & $39(17.6 \%)$ & $0(0 \%)$ & \\
\hline Stage 2 & $168(50.8 \%)$ & $129(58.4 \%)$ & $39(35.5 \%)$ & \\
\hline Stage 3 & $110(33.2 \%)$ & $39(17.6 \%)$ & $71(64.5 \%)$ & \\
\hline Enrollment non-renal APACHE III score & $54(43-71)$ & $53(41-69)$ & $58(45-82)$ & 0.017 \\
\hline
\end{tabular}

${ }^{a}$ As determined by retrospective analysis

protein 1 (CHI3L1), plasma cystatin C, urinary liver fatty acid binding protein (L-FABP), urinary kidney injury molecule-1 (KIM-1)] or with antibody pairs from R\&D Systems (Minneapolis, MN, USA) [glutathione $S$-transferase-pi (GST-pi), interleukin-18 (IL-18)]. Creatinine was measured by LabCorp (Burlington, North Carolina, USA) using the Jaffe method in serum collected at the same time as urine and plasma collected for biomarker measurements.

\section{Statistical analysis}

A sample size of at least 300 patients was selected to achieve a half-width of the $95 \% \mathrm{CI} \leq 0.05$ for the ROC AUC, providing a sufficiently narrow CI to assess biomarker efficacy. Continuous and categorical baseline variables were compared between endpoint negative and positive patients using the Wilcoxon rank-sum test and Fisher's exact test, respectively. Predictive ability of the biomarkers, including all combinations of two and three biomarkers (novel or previously described), for the 
primary endpoint of persistent severe AKI was assessed using the area under the receiver operating characteristic (ROC) curve. Confidence intervals of the area under the ROC curve (AUC) and pair-wise comparisons of AUC were calculated by the Delong method. Occurrence of MAKE $_{90}$ was compared across CCL14 tertiles using the Cochran-Armitage test. The cumulative incidence curves for the composite of RRT initiation or death were estimated by the Kaplan-Meier method, and log-rank test was used to compare the groups. Statistical analyses were performed using R 3.5.1. Two-sided $p$ values $<0.05$ were considered statistically significant. To examine whether CCL14 improved risk prediction beyond clinical variables alone, a reference multiple logistic regression model was constructed (Supplement) with those clinical variables significantly associated with the primary endpoint and further selected by a LASSO procedure. Integrated discrimination improvement (IDI) and category-free net reclassification improvement (cfNRI) were calculated with the "Hmisc" R package to assess the enhancement of risk prediction by the addition of CCL14 [15].

\section{Results}

364 patients were enrolled in the RUBY study, of whom 331 (91\%) were available for the primary analysis (Fig. 1). Approximately one-third (110 patients) of the analysis cohort met the primary endpoint of persistent stage 3 AKI, of whom 56 (51\%) received RRT and $14(13 \%)$ died prior to achieving 72 consecutive hours at stage 3.113 patients (34\%) met urine output criteria, 218 patients $(66 \%)$ met serum creatinine criteria, and $53(16 \%)$ patients met both criteria for stage 2-3 AKI at enrollment, with 45 (40\%), 103 (47\%), and 38 (72\%) meeting the primary endpoint, respectively. Patients who developed persistent stage 3 AKI had a lower BMI and were less likely to have a history of diabetes mellitus but had higher serum creatinine values, fluid balance, and APACHE III scores at enrollment compared to patients who did not develop persistent stage 3 AKI (Table 1). Patients who developed persistent stage 3 AKI were more likely to have stage 3 AKI at enrollment $(64.5 \%$ vs $17.6 \%$, $p<0.001$ ) which is consistent with higher enrollment levels of serum creatinine. Additionally, 128 (39\%) patients met the secondary MAKE $_{90}$ endpoint, of whom 108 $(84 \%)$ were deceased, $13(10 \%)$ had $\geq 25 \%$ loss in eGFR, and $7(5 \%)$ were on dialysis at $90( \pm 7)$ days.

Of the biomarkers tested in this study, urinary $\mathrm{C}-\mathrm{C}$ motif chemokine ligand 14 (CCL14) was most predictive of persistent stage 3 AKI with an AUC (95\% CI) of $0.83(0.78-0.87)$, which was significantly greater than the AUC values for the other biomarkers tested (Fig. 2 and Table S1). Urinary CHI3L1 [16], plasma cystatin
C, plasma proenkephalin, urinary NGAL, and urinary L-FABP had AUC values between 0.70 and 0.75 (Fig. 2). Combinations of CCL14 with the other biomarkers did not significantly improve the AUC, with the exception of plasma cystatin C (AUC increase $=0.028, p=0.04$ ) (Table S2). For all biomarkers associated with AKI, concentrations increased with AKI stage (Fig. 3). Among patients who did not persist at any stage of AKI, the urinary CCL14 concentrations within different comorbid conditions were similar suggesting urinary CCL14 elevations were specific to AKI persistence. Of note, the other AKI biomarkers in Fig. 3 showed substantial elevations in patients with some comorbid conditions even if they did not have persistent AKI.

Incidence of $\mathrm{MAKE}_{90}$ was $29 \%, 40 \%$, and $47 \%$, respectively, within the first, second, and third tertiles of CCL14 concentrations $(p=0.01)$. Mortality at 90 days also increased across CCL14 tertiles $(p=0.03)$, but the dialysis and eGFR loss components of $\mathrm{MAKE}_{90}$ did not significantly increase across CCL14 tertiles (Table S6). Cumulative incidence of the composite endpoint of RRT initiation or death within 90 days also increased across CCL14 tertiles $(p<0.001)$, approximately doubling from 32 to $63 \%$ for the first to third tertile (Fig. 4). Most events (91\%) occurred within the first 30 days after enrollment. The largest component of $\mathrm{MAKE}_{90}$ was mortality, which occurred in 108 (84\%) of patients.

Whether CCL14 enhances predictive ability over clinical variables was tested in a logistic regression model. Urinary CCL14 significantly improved risk prediction when added to a five-parameter clinical model for the

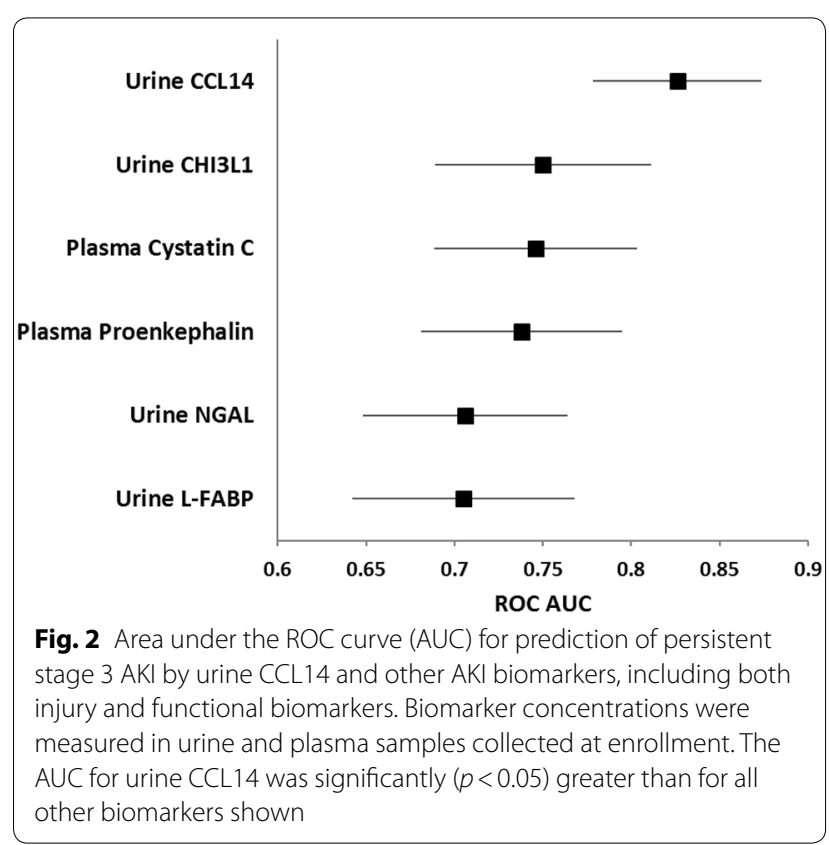


primary endpoint, using ROC AUC, integrated discrimination improvement (IDI), and category-free net reclassification improvement (cfNRI) analyses (Tables S3 and S4). All analyses showed significant enhancement by the addition of CCL14 which remained strongly associated with the primary endpoint.

A sensitivity analysis on the definition of AKI persistence demonstrated consistent performance of CCL14 across persistence durations of $24,48,72,96$, and $120 \mathrm{~h}$ and progression windows of 24,48 , and $72 \mathrm{~h}$ (Table S5). The ROC AUC (95\% CI) ranged from $0.79(0.74-0.84)$ for $24 \mathrm{~h}$ persistence and progression within $24 \mathrm{~h}$ to $0.84(0.79-0.88)$ for $48 \mathrm{~h}$ persistence and progression within $48 \mathrm{~h}$. Moreover, a sensitivity analysis in which the 53 patients retrospectively determined not to be at KDIGO stage 2-3 at enrollment (Table 1) were excluded showed nearly no change in the results for the primary endpoint [AUC $(95 \% \mathrm{CI})=0.81(0.76-$ $0.86)]$. Among the patients not at KDIGO stage $2-3$ at enrollment, $37(70 \%)$ decreased from stage $2-3$ by the time of enrollment, 10 (19\%) were determined to have a lower KDIGO stage after adjudication of baseline serum creatinine, and $6(11 \%)$ did not meet KDIGO criteria at any time.

\section{Discussion}

We have shown in a large international cohort of surgical and medical intensive care patients that several candidate molecules predict persistence of AKI, but that the best performing is a previously unknown candidate, urinary CCL14. Given this performance, we posit that CCL14 could be an important mediator of renal tissue damage and non-recovery. This is the first time such a finding has been described in AKI and the potential implications are considerable both for clinical management of patients with AKI and potential advancement of our understanding of the mechanism and potential therapeutic targets in AKI.

The results of the RUBY study have potential to be directly translatable to the clinical management of patients. A diagnostic test based on CCL14 which provides evidence of AKI persistence would aid in determining which patients may ultimately require RRT and which patients are likely to recover quickly and thus are unlikely to benefit from RRT initiation. Second, individuals highlighted as high risk for AKI persistence may be triaged to a referral center or to a higher level of care to mitigate potential complications of AKI, including emergent RRT. Third, identification of AKI persistence may allow for interventions to mitigate the important transition to CKD [5].

Our results may help to advance the understanding of the mechanism of AKI and in particular pathways related to tissue damage and non-recovery of renal function. AKI is associated with renal inflammation and during this process, circulating monocytes are recruited, become activated and differentiate into macrophages. Both glomerular and interstitial macrophage infiltration can be observed following injury and macrophages seem to play a diverse role in kidney damage and repair [17]. Indeed, in response to tissue injury, macrophages become activated with the functional state of the macrophage depending on the stage of tissue injury and repair. Therefore macrophages may contribute both to tissue injury and repair after AKI [18]. Such macrophage polarization is dictated by the immediate microenvironment and appears to play a critical role in the recovery of renal function after AKI.

CCL14 is a member of the chemokine family of small molecules that were initially recognized for roles in leukocyte chemotaxis and are implicated in tissue injury and repair processes. CCL14 binds with high affinity to the chemokine receptors CCR 1 and CCR5 and lower affinity to CCR3 [19]. CCL14 has been shown to be an important chemokine for monocyte/macrophage recruitment and is associated with pro-inflammatory chemotaxis in a variety of diseases including rheumatoid arthritis, multiple sclerosis, and lupus [20, 21]. Little is known regarding the role and nature of CCL14 in AKI, presumably because CCL14 is not expressed in mice and rats, so cannot be studied in pre-clinical models using these animal models. However, because macrophage recruitment and polarization are believed to play important roles in kidney tissue damage and development of persistent kidney dysfunction [17], a putative role for CCL14 can be proposed (Fig. 5). In addition, CCL14 has been shown to be an inflammatory marker identifying the risk of developing end-stage renal disease in diabetics [22].

This proposed mechanism may explain the association of elevated levels of CCL14 with persistence of severe AKI and non-recovery. The amount of CCL14 secreted

(See figure on next page.)

Fig. 3 Biomarker concentrations for different non-AKI acute and chronic conditions and by severity of persistent AKI for a urine CCL14, b urine CHI3L1, c plasma cystatin C, d plasma proenkephalin, e urine NGAL, and $\mathbf{f}$ urine L-FABP. Open boxes are for different acute and chronic conditions among patients who did not persist at any stage of AKI. Shaded boxes are for patients by persistent AKI stage. Box and whiskers show interquartile ranges and total observed ranges (censored by 1.5 times the box range), respectively 


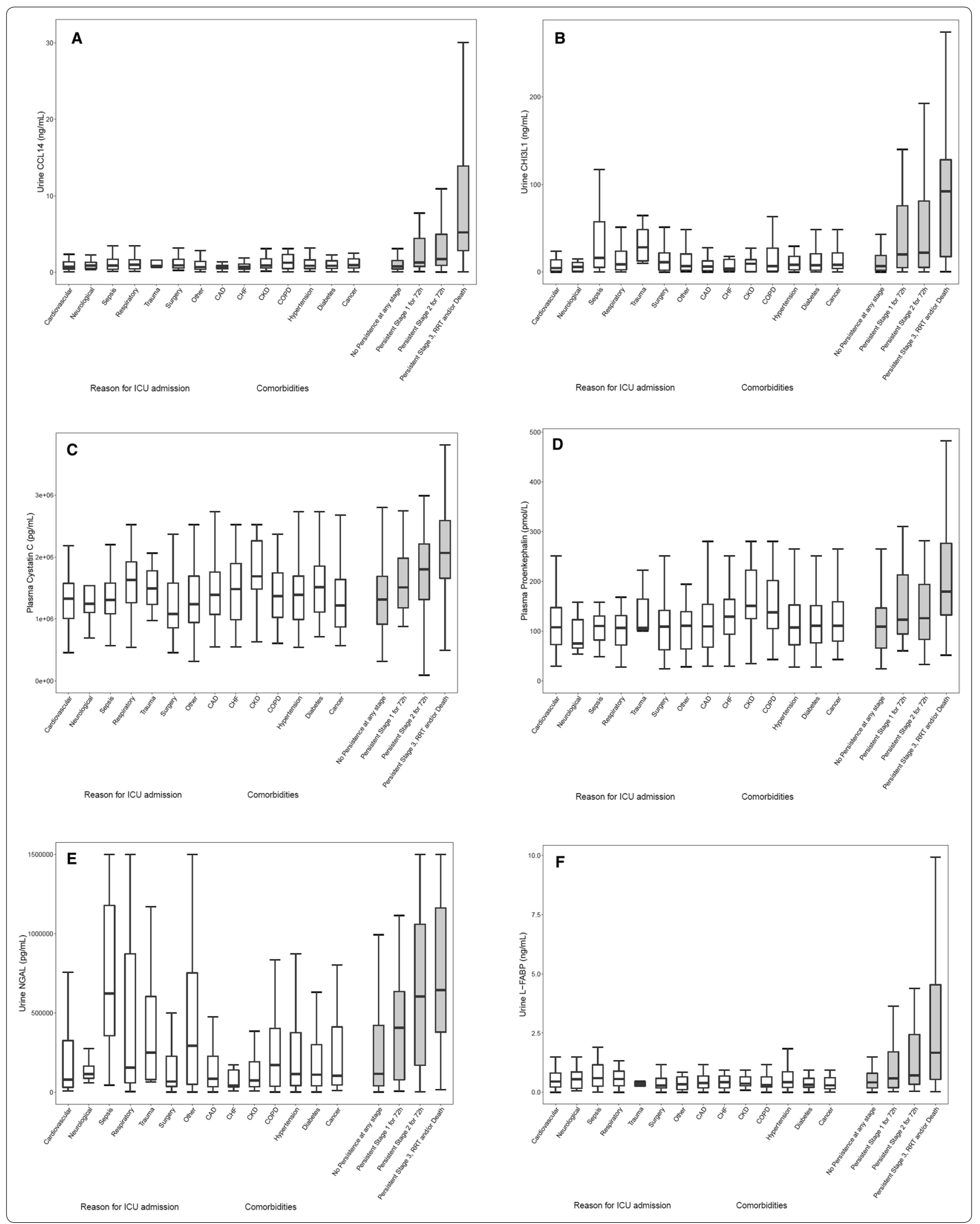




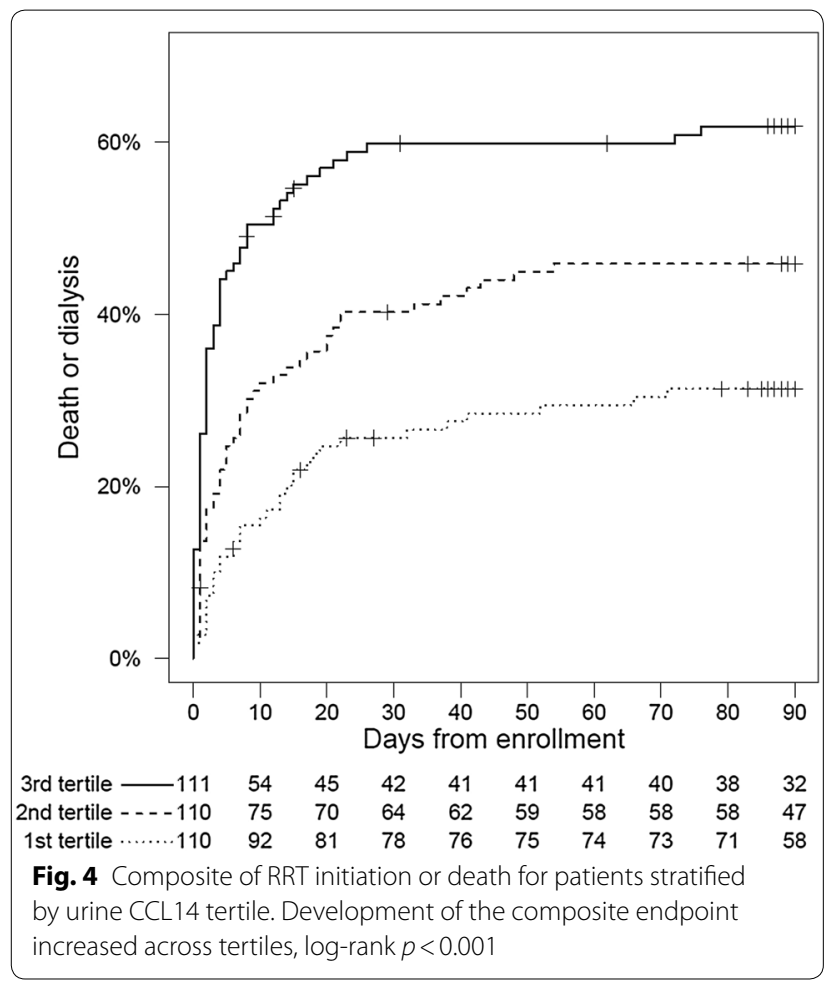

could reflect the extent of tissue damage. Moreover, higher levels of CCL14 may result in the recruitment of larger numbers of monocytes and T-cells which, in a proinflammatory injurious environment, can differentiate into $\mathrm{M}_{1}$ macrophages and Th1 cells that are pathogenic and can extend and magnify tissue damage. CCL14 could thus provide novel diagnostic and prognostic information that is important for managing patients with AKI, including decisions related to initiation of RRT. These assertions remain speculative and will require further investigation.

Our study has several strengths. First, this was an international multi-center trial that used the latest consensus guidelines to define the stage of AKI and persistent dysfunction $[5,10]$. Second, all investigators and study personnel were unaware of the biomarker test results which were batched and measured centrally. Third, we measured a broad range of candidate kidney biomarkers in this study. Our study also has limitations. For example, the type of AKI (nephrotoxic, inflammatory, versus septic) was not adjudicated thus performance of various biomarkers may differ in subsets of AKI. In addition, the secondary outcome variable, $\mathrm{MAKE}_{90}$, was largely driven by mortality and the study had low inclusion to screening ratio. Lastly, the study had a small proportion of patients who were misclassified at enrollment.

In summary, our findings demonstrate that an elevation in urinary CCL14 predicts the persistence/progression of AKI in a large cohort of mixed critically ill patients with severe AKI. Consistent with pre-clinical studies, chemokine signaling and macrophage trafficking appear to play an important role in renal repair and recovery. Discovery of CCL14 as predictor of persistent 
A $\mathrm{TNF}_{\alpha}$ and other inflammatory mediators CCL14

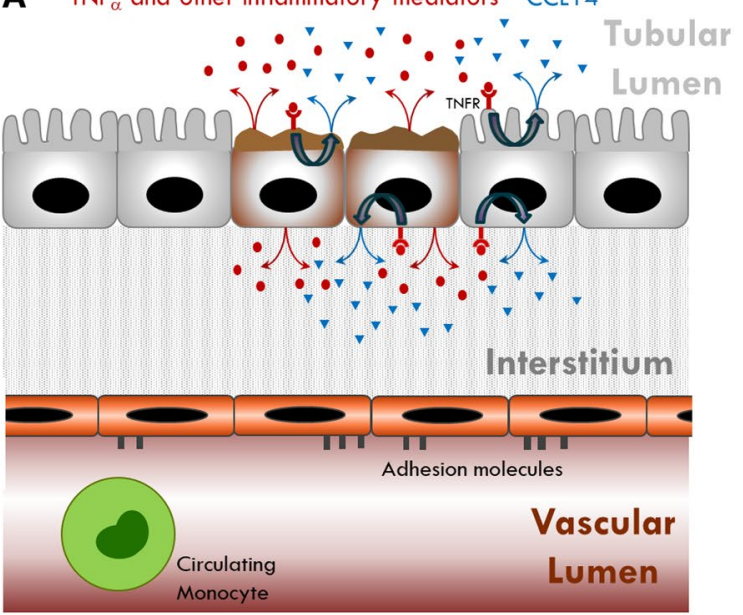

CCLI 4

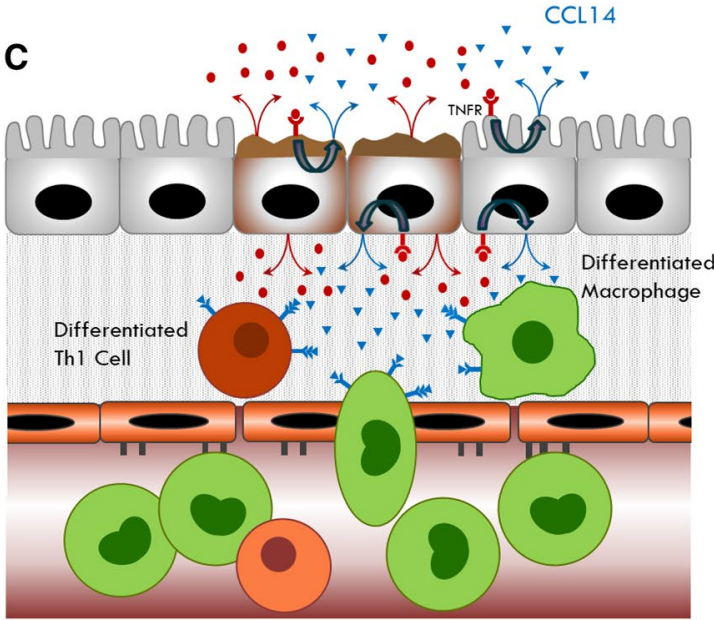

B

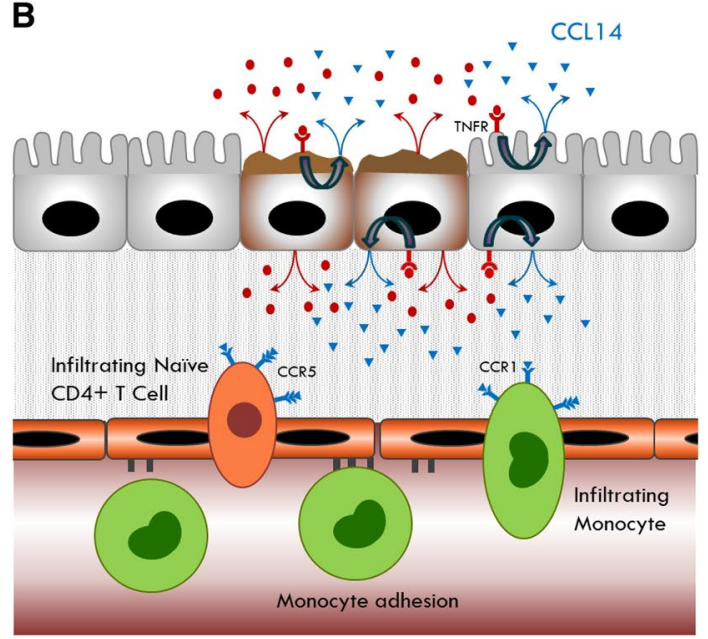

Fig. 5 Proposed CCL14 mechanism. a TNFa and other inflammatory mediators are released from injured epithelium and bind to TNF receptors, leading to release of CCL14 from tubular epithelial cells. b Binding of CCL14 to CCR1 and CCR5 receptors on monocytes and T cells induces chemotaxis towards site of injury. c Monocytes differentiate into macrophages and naïve T cells differentiate into proinflammatory Th1 cells

AKI and thus, renal non-recovery may not only directly affect treatment options in the acute phase but offers the potential to identify new therapeutic approaches to AKI.

\section{Electronic supplementary material}

The online version of this article (https://doi.org/10.1007/s00134-019-05919-0) contains supplementary material, which is available to authorized users.

\section{Author details}

${ }^{1}$ Ghent University Hospital, Ghent University, Ghent, Belgium. ${ }^{2}$ Department of Medicine, University of Florida, Gainesville, FL, USA. ${ }^{3}$ Department of Critical Care Medicine, University of Pittsburgh School of Medicine, Pittsburgh, PA, USA. ${ }^{4}$ Allegheny General Hospital, Pittsburgh, PA, USA. ${ }^{5}$ King's College London, Guy's \& St Thomas' Hospital, London, UK. ${ }^{6}$ MVZ Diaverum Am Neuen Garten, Potsdam, Germany. ${ }^{7}$ Medizinische Fakultät, Otto-Von-Guericke Universität Magdeburg, Magdeburg, Germany. ${ }^{8}$ University Hospital Frankfurt, Goethe University, Frankfurt, Germany. ${ }^{9}$ Department of Medicine, Pulmonary and Critical Care Division, Northwestern University Feinberg School of Medicine, Chicago, IL, USA. ${ }^{10}$ Division of Nephrology, Department of Medicine, University of Michigan, Ann Arbor, MI, USA. ${ }^{11}$ Rutgers-Robert Wood Johnson
Medical School, New Brunswick, NJ 08901, USA. ${ }^{12}$ Department of Emergency Medicine, Vanderbilt University Medical Center, Nashville, TN, USA. ${ }^{13}$ Section of Nephrology, Department of Medicine, University of Chicago, Chicago, IL, USA. ${ }^{14}$ University Hospital Brussels, Brussels, Belgium. ${ }^{15}$ Royal London Hospital, Barts Health NHS Trust, London, UK. ${ }^{16}$ Division of Intensive Care and Emergency Medicine, Department of Internal Medicine, Medical University Innsbruck, Innsbruck, Austria. ${ }^{17}$ Department of Clinical \& Experimental Medicine, Faculty of Health Sciences, University of Surrey, Guildford, UK.

${ }^{18}$ Astute Medical, Inc, San Diego, CA, USA. ${ }^{19}$ Department of Critical Care Medicine, Center for Critical Care Nephrology, University of Pittsburgh, Pittsburgh, PA, USA. ${ }^{20}$ Department of Medicine, Veterans Affairs Medical Center, 3350 La Jolla Village Dr, San Diego, CA 92161, USA.

\section{Acknowledgements}

RUBY Investigators: Eric Hoste (Ghent University Hospital, Ghent University Ghent, Belgium), Azra Bihorac (Department of Medicine, University of Florida, Gainesville, FL, USA), Ali Al-Khafaji (Department of Critical Care Medicine, University of Pittsburgh School of Medicine, Pittsburgh, PA, USA), Luis M. Ortega (Allegheny General Hospital, Pittsburgh, PA, USA), Marlies Ostermann (King's College London, Guy's \& St Thomas' Hospital, London, UK), Michael Haase (MVZ Diaverum Am Neuen Garten, Potsdam; Medizinische Fakultät, Otto-vonGuericke Universität Magdeburg, Magdeburg, Germany), Kai Zacharowski 
(University Hospital Frankfurt, Goethe University, Frankfurt, Germany), Richard Wunderink (Department of Medicine, Pulmonary and Critical Care Division, Northwestern University Feinberg School of Medicine, Chicago IL, USA), Michael Heung (Division of Nephrology, Department of Medicine University of Michigan, Ann Arbor, MI), Kyle Gunnerson (Department of Emergency Medicine, MCIRCC, Michigan Medicine, Ann Arbor, MI), Matthew Lissauer (Rutgers-Robert Wood Johnson Medical School, New Brunswick, NJ 08901), Daniel Herr (Dept of internal medicine. University of Maryland), Wesley H. Self (Department of Emergency Medicine, Vanderbilt University Medical Center, Nashville, Tennessee, USA), Jay L. Koyner (Section of Nephrology, Department of Medicine, University of Chicago, Chicago IL, USA), Patrick M. Honore (Univeristy Hospital Brussels, Brussels, Belgium), John R. Prowle (Royal London Hospital, Barts Health NHS Trust. London, UK), Danielle Davison (Department of Anesthesiology and Critical Care Medicine, The George Washington University, Washington, D.C. USA), Antonio Artigas (Critical Center, Corporación Sanitaria Universitaria Parc Tauli, CIBER Enfermedades Respiratorias, Autonomous University of Barcelona), Michael Joannidis (Division of Intensive Care and Emergency Medicine, Department of Internal Medicine, Medical University Innsbruck, Innsbruck, Austria), Rebecca Schroeder (Duke University School of Medicine, Durham, NC, USA), Sevag Demirjian (Department of Nephrology, Cleveland Clinic Foundation, Cleveland, OH, USA), Lui G. Forni (Department of Clinical \& Experimental Medicine, Faculty of Health Sciences, University of Surrey, Guildford, UK), Luke Hodgson (Worthing Hospital, Worthing, U.K), Scott T. Wilber (Northeast Ohio Medical University, Rootstown, Ohio), Jennifer A. Frey (Summa Health System, Akron, Ohio, USA), lan Reilly (Scripps Medical Center, San Diego, CA, USA), Jing Shi (Walker Biosciences, Carlsbad, CA, USA), J. Patrick Kampf (Astute Medical, Inc., San Diego, CA, USA), Thomas Kwan (Astute Medical, Inc., San Diego, CA, USA), Paul McPherson (Astute Medical, Inc., San Diego, CA, USA), John A. Kellum (Center for Critical Care Nephrology, Department of Critical Care Medicine, University of Pittsburgh, Pittsburgh PA, USA), and Lakhmir S. Chawla (Department of Medicine, Veterans Affairs Medical Center, San Diego, CA, USA)

\section{Author contributions}

EH helped design the study, acquired study data, analyzed the study results, helped draft the manuscript, made critical revisions and provided final approval; AB, AAK, LO, MH, KZ, RW, MH, KG, ML, DH, WS, JK, PMH, JRP, DD, AA, $\mathrm{MJ}, \mathrm{RS}, \mathrm{SD}, \mathrm{LH}, \mathrm{SW}$, and JF acquired study data, analyzed the study results, and made critical revisions and provided final approval; MO and LGF acquired study data, analyzed the study results, drafted the manuscript, made critical revisions and provided final approval; IR, JS, JPK, TK, and PM analyzed study results, made critical revisions and provided final approval; JAK and LSC designed the trial, analyzed study results, drafted the manuscript, made critical revisions and provided final approval.

\section{Funding}

Astute Medical, Inc.

\section{Compliance with ethical standards}

\section{Conflicts of interest}

Jing Shi, John Kellum, Jay Koyner, and Lakhmir Chawla report receiving consulting fees from Astute Medical. Paul McPherson, Patrick Kampf, and Thomas Kwan are employees of Astute Medical, a bioMérieux subsidiary. Marlies Ostermann reports receiving consulting fees from bioMérieux. Lui Forni reports receiving consulting fees from Ortho Clinical Diagnostics. John Prowle reports receiving research support from bioMérieux. Michael Haase reports receiving lecture fees and travel cost reimbursement from Abbott Diagnostics, Astute Medical, FastBiomedical, and Alere.

\section{Ethical approval}

The RUBY study was approved by the Western Institutional Review Board (Puyallup, WA) and by individual investigational review boards or ethics committees as required by each enrolling site.

\section{Informed consent}

Informed consent was obtained from all individual participants included in the study or their legally authorized representatives.

\section{Open Access}

This article is licensed under a Creative Commons Attribution-NonCommercial 4.0 International License, which permits any non-commercial use, sharing, adaptation, distribution and reproduction in any medium or format, as long as you give appropriate credit to the original author(s) and the source, provide a link to the Creative Commons licence, and indicate if changes were made. The images or other third party material in this article are included in the article's Creative Commons licence, unless indicated otherwise in a credit line to the material. If material is not included in the article's Creative Commons licence and your intended use is not permitted by statutory regulation or exceeds the permitted use, you will need to obtain permission directly from the copyright holder.To view a copy of this licence, visit http://creativecommons.org/licen ses/by-nc/4.0/.

\section{Publisher's Note}

Springer Nature remains neutral with regard to jurisdictional claims in published maps and institutional affiliations.

Received: 29 August 2019 Accepted: 26 December 2019

Published online: 6 February 2020

\section{References}

1. Koyner JL, Shaw AD, Chawla LS, Hoste EA, Bihorac A, Kashani K, Haase M, Shi J, Kellum JA, Sapphire I (2015) Tissue inhibitor metalloproteinase-2 (TIMP-2)|GF-binding protein-7 (IGFBP7) levels are associated with adverse long-term outcomes in patients with AKI. J Am Soc Nephrol 26(7):17471754. https://doi.org/10.1681/ASN.2014060556

2. Mehta S, Chauhan K, Patel A, Patel S, Pinotti R, Nadkarni GN, Parikh CR, Coca SG (2018) The prognostic importance of duration of AKI: a systematic review and meta-analysis. BMC Nephrol 19(1):91. https://doi. org/10.1186/s12882-018-0876-7

3. Perinel $S$, Vincent F, Lautrette A, Dellamonica J, Mariat C, Zeni F, Cohen Y, Tardy B, Souweine B, Darmon M (2015) Transient and persistent acute kidney injury and the risk of hospital mortality in critically ill patients: results of a multicenter cohort study. Crit Care Med 43(8):e269-275. https //doi.org/10.1097/CCM.0000000000001077

4. Kellum JA, Sileanu FE, Bihorac A, Hoste EA, Chawla LS (2017) Recovery after acute kidney injury. Am J Respir Crit Care Med 195(6):784-791. https ://doi.org/10.1164/rccm.201604-07990C

5. Chawla LS, Bellomo R, Bihorac A, Goldstein SL, Siew ED, Bagshaw SM, Bittleman D, Cruz D, Endre Z, Fitzgerald RL, Forni L, Kane-Gill SL, Hoste E, Koyner J, Liu KD, Macedo E, Mehta R, Murray P, Nadim M, Ostermann M, Palevsky PM, Pannu N, Rosner M, Wald R, Zarbock A, Ronco C, Kellum JA, Acute Disease Quality Initiative W (2017) Acute kidney disease and renal recovery: consensus report of the Acute Disease Quality Initiative (ADQI) 16 Workgroup. Nat Rev Nephrol 13(4):241-257. https://doi.org/10.1038/ nrneph.2017.2

6. Chawla LS, Eggers PW, Star RA, Kimmel PL (2014) Acute kidney injury and chronic kidney disease as interconnected syndromes. N Engl J Med 371(1):58-66. https://doi.org/10.1056/NEJMra1214243

7. Bagshaw SM, Lamontagne F, Joannidis M, Wald R (2016) When to start renal replacement therapy in critically ill patients with acute kidney injury: comment on AKIKI and ELAIN. Crit Care 20(1):245. https://doi. org/10.1186/s13054-016-1424-0

8. Forni LG, Joannidis M (2019) IDEAL timing of renal replacement therapy in critical care. Nat Rev Nephrol 15(1):5-6. https://doi.org/10.1038/s4158 1-018-0088-1

9. Ferenbach DA, Bonventre JV (2015) Mechanisms of maladaptive repair after AKI leading to accelerated kidney ageing and CKD. Nat Rev Nephrol 11(5):264-276. https://doi.org/10.1038/nrneph.2015.3 
10. Kidney Disease: Improving Global Outcomes (KDIGO) Acute Kidney Injury Work Group (2012) KDIGO clinical practice guideline for acute kidney injury. Kidney Int Suppl 17:1-138

11. Knottnerus JA, Muris JW (2003) Assessment of the accuracy of diagnostic tests: the cross-sectional study. J Clin Epidemiol 56(11):1118-1128

12. Gaudry S, Hajage D, Schortgen F, Martin-Lefevre L, Pons B, Boulet E, Boyer A, Chevrel G, Lerolle N, Carpentier D, de Prost N, Lautrette A, Bretagnol A, Mayaux J, Nseir S, Megarbane B, Thirion M, Forel JM, Maizel J, Yonis H, Markowicz P, Thiery G, Tubach F, Ricard JD, Dreyfuss D, Group AS (2016) Initiation strategies for renal-replacement therapy in the intensive care unit. N Engl J Med 375(2):122-133. https://doi.org/10.1056/NEJMoa1603 017

13. Billings FTT, Shaw AD (2014) Clinical trial endpoints in acute kidney injury. Nephron Clin Pract 127(1-4):89-93. https://doi.org/10.1159/000363725

14. Kashani K, Al-Khafaji A, Ardiles T, Artigas A, Bagshaw SM, Bell M, Bihorac A, Birkhahn R, Cely CM, Chawla LS, Davison DL, Feldkamp T, Forni LG, Gong MN, Gunnerson KJ, Haase M, Hackett J, Honore PM, Hoste EA, JoannesBoyau O, Joannidis M, Kim P, Koyner JL, Laskowitz DT, Lissauer ME, Marx G, McCullough PA, Mullaney S, Ostermann M, Rimmele T, Shapiro NI, Shaw AD, Shi J, Sprague AM, Vincent JL, Vinsonneau C, Wagner L, Walker MG, Wilkerson RG, Zacharowski K, Kellum JA (2013) Discovery and validation of cell cycle arrest biomarkers in human acute kidney injury. Crit Care 17(1):R25. https://doi.org/10.1186/cc12503

15. Harrell FE (2018) Hmisc: harrell miscellaneous. R package version 4.1-1. https://CRAN.R-project.org/package=Hmisc. Accessed 22 Feb 2019

16. De Loor J, Decruyenaere J, Demeyere K, Nuytinck L, Hoste EA, Meyer E (2016) Urinary chitinase 3-like protein 1 for early diagnosis of acute kidney injury: a prospective cohort study in adult critically ill patients. Crit Care 20:38. https://doi.org/10.1186/s13054-016-1192-x

17. Meng XM, Tang PM, Li J, Lan HY (2015) Macrophage phenotype in kidney injury and repair. Kidney Dis (Basel) 1(2):138-146. https://doi. org/10.1159/000431214

18. Huen SC, Cantley LG (2017) Macrophages in renal injury and repair. Annu Rev Physiol 79:449-469. https://doi.org/10.1146/annurev-physiol-02251 6-034219

19. Detheux M, Standker L, Vakili J, Munch J, Forssmann U, Adermann K, Pohlmann S, Vassart G, Kirchhoff F, Parmentier M, Forssmann WG (2000) Natural proteolytic processing of hemofiltrate CC chemokine 1 generates a potent CC chemokine receptor (CCR) 1 and CCR5 agonist with anti-HIV properties. J Exp Med 192(10):1501-1508

20. Rump L, Mattey DL, Kehoe O, Middleton J (2017) An initial investigation into endothelial CC chemokine expression in the human rheumatoid synovium. Cytokine 97:133-140. https://doi.org/10.1016/j. cyto.2017.05.023

21. Vyshkina T, Sylvester A, Sadiq S, Bonilla E, Perl A, Kalman B (2008) CCL genes in multiple sclerosis and systemic lupus erythematosus. J Neuroimmunol 200(1-2):145-152. https://doi.org/10.1016/j.jneuroim.2008.05.016

22. Niewczas MA, Pavkov ME, Skupien J, Smiles A, Md Dom ZI, Wilson JM, Park J, Nair V, Schlafly A, Saulnier PJ, Satake E, Simeone CA, Shah H, Qiu C, Looker HC, Fiorina P, Ware CF, Sun JK, Doria A, Kretzler M, Susztak K, Duffin KL, Nelson RG, Krolewski AS (2019) A signature of circulating inflammatory proteins and development of end-stage renal disease in diabetes. Nat Med 25(5):805-813. https://doi.org/10.1038/s41591-019-0415-5 